\title{
Visualizing Urban Microclimate and Quantifying its Impact on Building Energy Use in San Francisco
}

\author{
Tianzhen Hong \\ Lawrence Berkeley National Laboratory \\ thong@lbl.gov
}

\author{
Yujie Xu \\ Carnegie Mellon University \\ yujiex@andrew.cmu.edu
}

\author{
Wanni Zhang \\ Lawrence Berkeley National Laboratory Lawrence Berkeley National Laboratory \\ wannizhang@lbl.gov \\ Xuan Luo \\ xuanluo@lbl.gov
}

\author{
Kaiyu Sun \\ Lawrence Berkeley National Laboratory \\ ksun@lbl.gov
}

\begin{abstract}
Weather data at nearby airports are usually used in building energy simulation to estimate energy use in buildings or evaluate building design or retrofit options. However, due to urbanization and geography characteristics, local weather conditions can differ significantly from those at airports. This study presents the visualization of 10-year hourly weather data measured at 27 sites in San Francisco, aiming to provide insights into the urban microclimate and urban heat island effect in San Francisco and how they evolve during the recent decade. The 10-year weather data are used in building energy simulations to investigate its influence on energy use and electrical peak demand, which informs the city's policy making on building energy efficiency and resilience. The visualization feature is implemented in CityBES, an open web-based data and computing platform for urban building energy research.
\end{abstract}

\section{CCS CONCEPTS}

- Human-centered computing $\rightarrow$ Visualization

- Human-centered computing $\rightarrow$ Heat maps

\section{KEYWORDS}

Urban microclimate, visualization, CityBES, San Francisco, building energy use, urban heat island

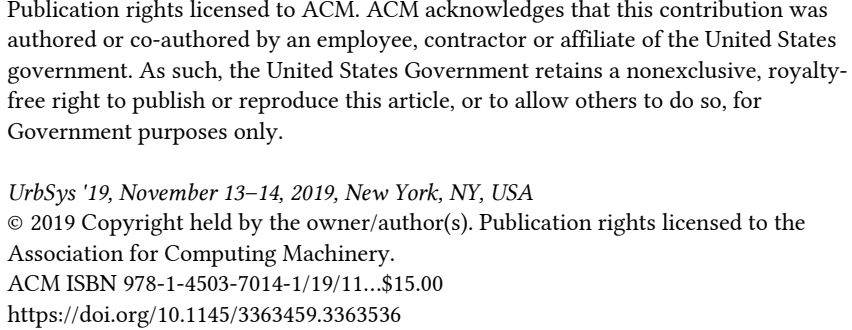

Publication rights licensed to ACM. ACM acknowledges that this contribution was authored or co-authored by an employee, contractor or affiliate of the United States government. As such, the United States Government retains a nonexclusive, royaltyfree right to publish or reproduce this article, or to allow others to do so, for Government purposes only.

UrbSys '19, November 13-14, 2019, New York, NY, USA

(c) 2019 Copyright held by the owner/author(s). Publication rights licensed to the Association for Computing Machinery.

ACM ISBN 978-1-4503-7014-1/19/11...\$15.00

https://doi.org/10.1145/3363459.3363536

\section{ACM Reference format:}

Tianzhen Hong, Yujie Xu, Kaiyu Sun, Wanni Zhang and Xuan Luo. 2019. Visualizing urban microclimate and quantifying its impact on building energy use in San Francisco. In Proceedings of 6th ACM International Conference on Systems for Energy-Efficient Buildings, Cities, and Transportation (BuildSys 2019). ACM, New York, NY, USA, 5 pages. https://doi.org/10.1145/3363459.3363536

\section{INTRODUCTION}

San Francisco is a coastal city in Northern California. It has a mild climate $3 \mathrm{C}$ and an area of $121.4 \mathrm{~km}^{2}$. Even though the city is not large geographically, its local climate can vary significantly across the city. Such microclimate variations have strong implications on building energy demand and outdoor thermal environment. We analyzed 10-year hourly weather data measured at 27 weather stations across the city and visualized the results using maps with the CityBES tool to reveal microclimate spatial patterns and the temporal trends. We also use building energy modeling to quantify the urban microclimate impact on building energy demand using local weather data at 13 sites. Here the impact refers to the same building in different locations of San Francisco will have different energy uses and peak demands due to local microclimate conditions. These results can inform the city's policy on the urban environment, building energy planning, as well as resilience issues due to extreme weather events such as heatwaves.

\section{VISUALIZATION OF SAN FRANCISCO LOCAL CLIMATE}

We developed a web-based map interface to visualize the spatial variation and temporal trends of San Francisco local climate. It is part of the City Building Energy Saver (CityBES) platform [1].

\subsection{Data Source and Data Cleaning}

2008 to 2017 hourly weather data were acquired from White Box Technologies [2]. The data set consists of 149 weather files in EnergyPlus epw format, each with one-year duration, containing 22 weather variables. The format and detailed variable descriptions follow section 2.9.1 in [3]. Among the files we received, seven of them have non-matching weather station IDs inside the weather file, and in the file name. This makes it difficult to locate the associated stations for those files. Mis-placing weather station data will impair the accuracy of the spatial interpolation step, thus we removed them from the data set. The second cleaning step involves dropping measurements with invalid data range. In this step, we removed three records with 
negative relative humidity values. After cleaning, we selected four variables to visualize in the interface: dry bulb temperature $\left({ }^{\circ} \mathrm{F}\right)$, relative humidity (\%), global horizontal radiation $\left(\mathrm{Wh} / \mathrm{m}^{2}\right)$, and wind speed $(\mathrm{m} / \mathrm{s})$. There are 27 weather stations in our cleaned data set. Figure 1 shows the location and start and end year of each weather station.

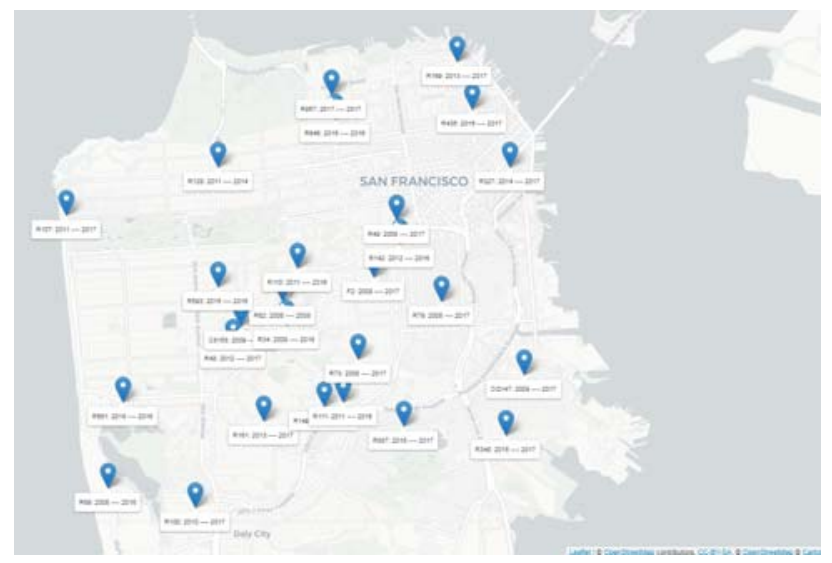

Figure 1: Weather Stations in San Francisco

In addition to the four direct measurements, the interface also displays four derived weather metrics: heating degree-day (HDD), cooling degree-day (CDD), heat index, and urban heat island index (UHII). HDD and CDD could inform urban planners and policy makers about the potential heating and cooling loads at various locations and at different times of the year. Heat index and UHII could assist policy analysis of adaptation strategies to short-term heat waves, and long-term climate change. The calculations of the derived metrics are described as follows.

2.1.1 HDD and CDD. Degree-days are often used for rough estimation of the heating or cooling load. Degree-days for a certain period $P$ (e.g., a month or a year) are computed as the accumulated difference between the mean daily temperature and some base temperature (Equation 1 and 2). Here we use $65^{\circ} \mathrm{F}$ as the base temperature for heating degree day, and $50^{\circ} \mathrm{F}$ for cooling degree day, consistent with the practice of ASHRAE handbooks.

$\mathrm{HDD}_{P}=\sum_{i \in P} \max \left(0,65-T_{\mathrm{i}, \text { mean }}\right)$

$\mathrm{CDD}_{P}=\sum_{i \in P} \max \left(0, T_{\mathrm{i}, \text { mean }}-50\right)$

$T_{\mathrm{i} \text {,mean }}$ is the average of the daily max and the daily min temperature of day $i$ in the period $P$.

2.1.2 Heat Index (HI) This metric reflect the hotness considering both temperature and relative humidity. The calculation follows the NOAA method [4], where the Steadman's formula is applied first. For high HI cases (higher than 80), the Rothfusz formula with certain adjustments is applied.

2.1.3 Urban Heat Island Index (UHII) Taha and Freed developed a UHII metric [5]. A UHII is calculated for each census tract as the accumulated hourly temperature difference between the urban and the non-urban areas within the census tract. We modified this definition and developed our own UHII metric to reflect more detailed spatial heterogeneity. In our definition, we put grids over the San Francisco area and compute a UHII for each grid cell (a $30 \mathrm{~m} \times 30 \mathrm{~m}$ region). We used the same reference non-urban-area temperature for all regions (pixels), as opposed to the approach of Taha and Freed [5] where each region (census tract) has its own un-urban area reference temperature. Equation 3 shows our definition of urban heat island index for pixel $\mathrm{p}$ for duration $\mathrm{D}$, $U H I I_{p D}$

$$
\mathrm{UHII}_{p D}=\sum_{h \in D} \mathbb{1}[\operatorname{urban}(p)] \cdot\left(\max \left(0, T_{p, h}-\overline{T_{u h}}\right)\right)
$$

$\mathbb{I}[\operatorname{urban}(p)]$ is an indicator variable of whether pixel $\mathrm{p}$ is of an urban land use type. $\overline{T_{u h}}$ is the average temperature of all unurban pixels in San Francisco. We used the 2011 National Land Cover Database (NLCD) raster file [6] to classify whether a pixel on the map is urban or non-urban. We follow [5] and label category 21 to 24 (the developed land use types) as urban area, and the rest as non-urban area. The NLCD data is retrieved with the R package, FedData [7].
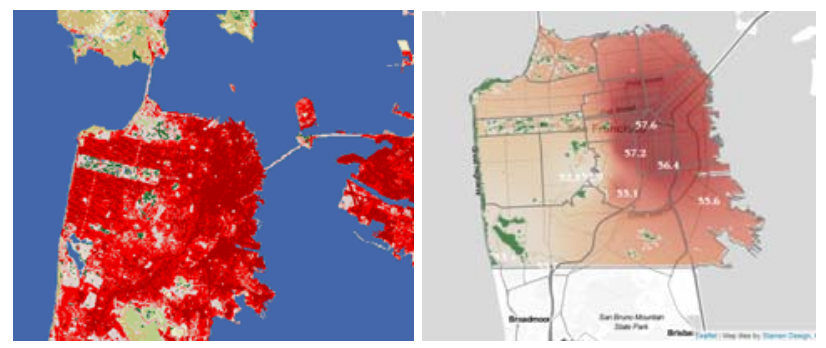

Figure 2: Land use pattern from NLCD (left), urban heat island index for April 2008 (right)

\subsection{Interface Design}

The map interface displays the space-time patterns of various weather metrics in San Francisco, with a 2D map view, a time slider navigating through different snapshots of the map view, and some data summary charts.

The spatial heterogeneity of each weather metric is presented as a heat map overlaid on top of the San Francisco regional map. To provide some spatial context, we overlaid the San Francisco planning district shape. The heat map of each weather variable is generated for each time stamp with an inverse distance weighted spatial interpolation of measurements at each weather station. A button on the bottom right of the map window (component 10) toggles a close-up view of local weather conditions around some building portfolio (Figure 2). Exact measurements at each weather station are shown as white labels on the map. Users can navigate through different time stamps with a time slider at the bottom of the interface. 

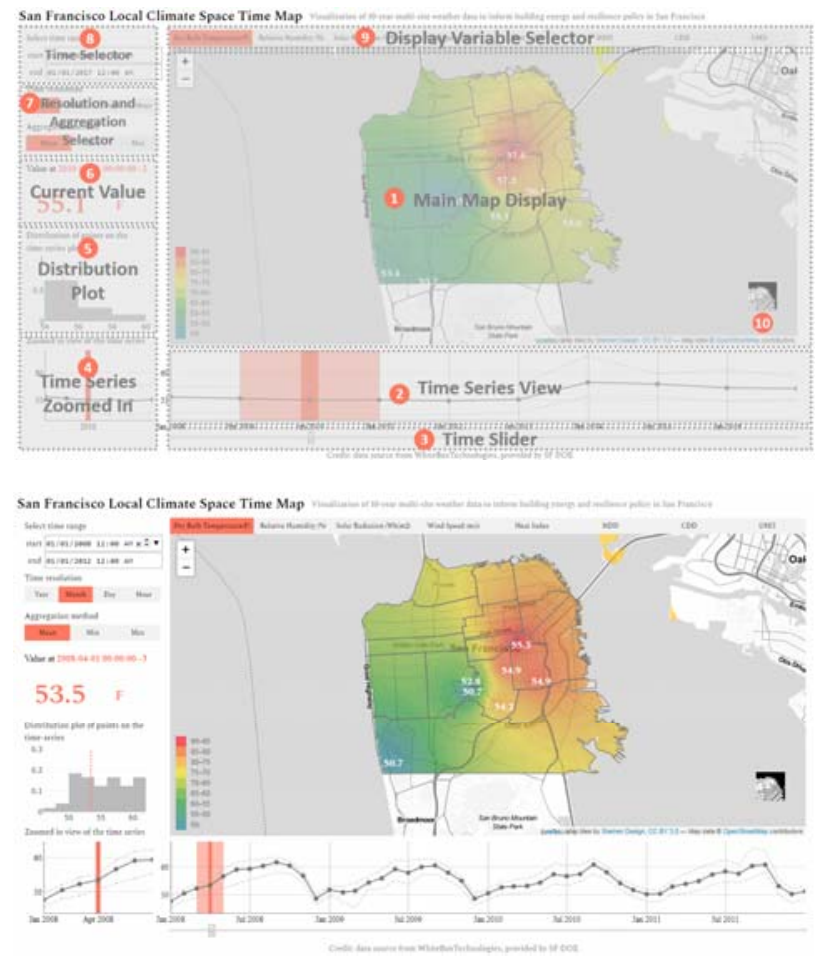

Figure 3: Components of the Interface (top), a screenshot of the interface (bottom)

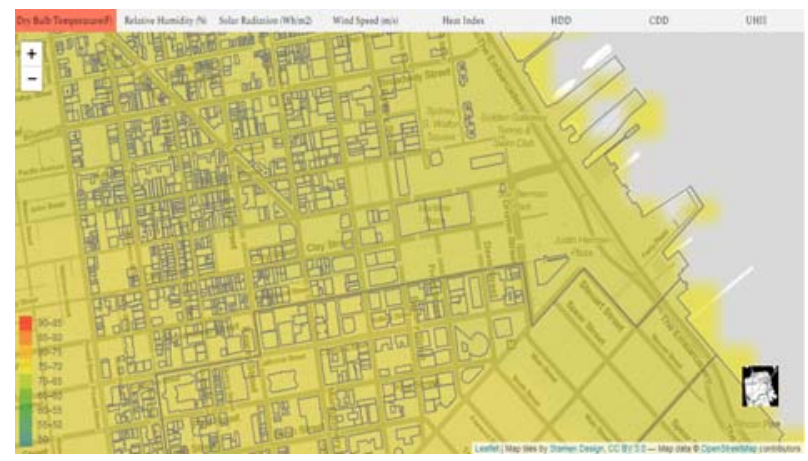

Figure 4: Spatial Close-up View

The temporal trend is shown as time-series plots (component 2 and 4). The plot below the map window shows the spatialtemporal aggregation (the solid line) and the spatial extremes (the min and max of the color-coded values displayed on the map, shown with two dashed lines) of the temporal aggregation. A histogram to the left of the map shows the distribution of the spatial-temporal aggregation for the displayed period, with the value of the current time step marked as a red dashed line.

Weather variables are anticipated to have strong seasonality at various temporal scales, thus we provided several temporal resolutions, and temporal aggregation options (component 7). For example, when the "year" resolution and the "mean" aggregation method are chosen, weather station data are aggregated to annual averages. Then a heat map is generated for each year with spatial interpolation of the annual average weather station data.

\subsection{Implementation}

$\mathrm{R}$ is used for data cleaning, processing, image and data file (CSV or JSON) generation. The heat maps are png images generated using $\mathrm{R}$ raster package [8], gstat package [9], and sf package [10]. The interface is written in HTML and JavaScript. We use Leaflet [11] to create the map view and load the heat map image onto the regional map. The time-series plots are created with dygraphs [12]. The histogram is produced with Plotly [13].

\subsection{Example use cases}

The visualization tool reveals some interesting patterns in the weather data. We present three use cases as examples:

1. With the daily or hourly view, users could identify certain historical weather events. For example, in the 2017 daily drybulb temperature display (Figure 5), we could observe that the timing (September $\left.1^{\mathrm{st}}\right)$ and the magnitude $\left(101.4^{\circ} \mathrm{F}\right)$ of the heatwave from the time-series plot, and the current-value label (component 5). The distribution plot further demonstrated that the temperature at the current time stamp is at the right end of the distribution.

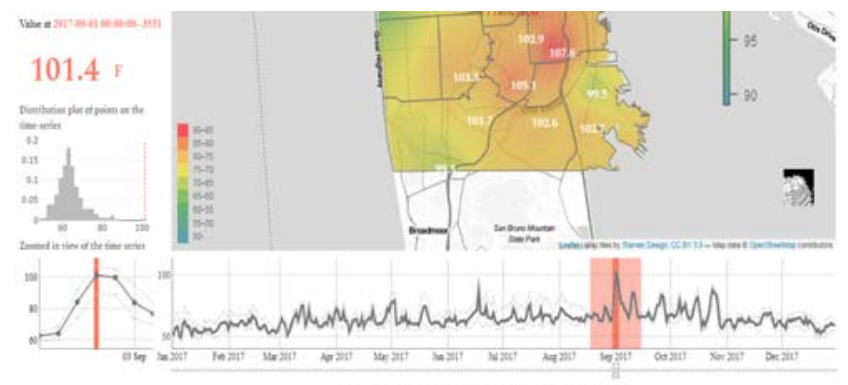

Figure 5: Components of the Interface (top), a screenshot of the interface (bottom)

2. With the monthly views, users could spot seasonality of certain variables. For example, we could observe strong seasonal patterns in temperature, solar radiation, and wind speed, while relative humidity is relatively stable - not seasonal.

3. Using the time-series plot (component 2), users could identify the overall level of spatial heterogeneity of a variable (the distance between the two dashed lines). With the map view, user could locate the spatial extreme positions. For example, in the daily mean relative humidity view, we notice substantial spatial variations where the maximum is almost twice the minimum. The map view shows the highest values are close to the coastal area, and the lowest values appear in inland regions. 


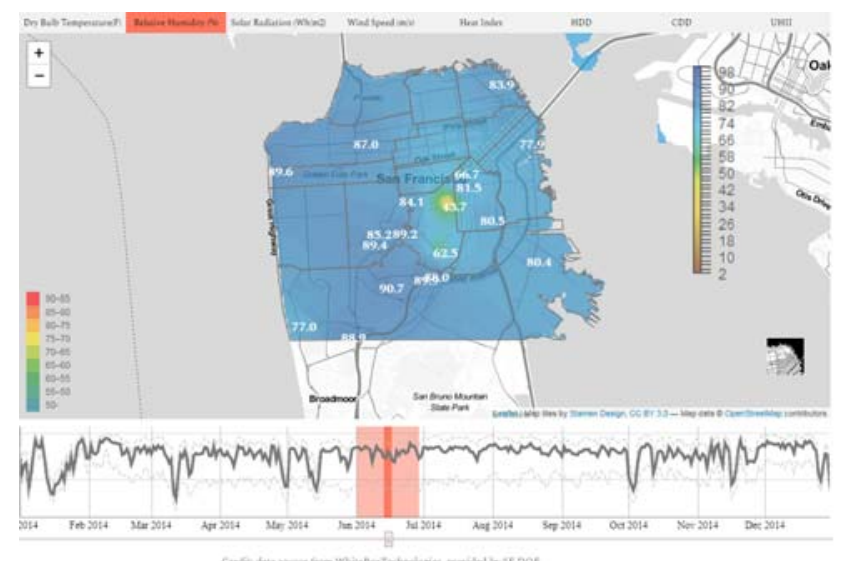

Figure 6: Spatial heterogeneity of relative humidity

\section{MICROCLIMATE IMPACT ON BUILDING ENERGY USE}

\subsection{Simulation description}

A simulation study was performed to evaluate the impact of microclimate on building energy use. The weather data in 2017 at 13 stations were applied to four DOE prototype building energy models [15], covering two building types (the large office and the large hotel) and two vintages (2004 and 2013). EnergyPlus is used as the building performance simulation tool in this study to calculate the annual energy use and peak demand of the four building models using the 10-year weather files at the 13 weather stations. EnergyPlus is an open-source program that models heating, ventilation, cooling, lighting, water use, renewable energy generation, and other building energy flows [16] and is the flagship building simulation engine supported by the United States Department of Energy (DOE).

\subsection{Results}

Figures 7-10 illustrate the box-whisker plots, which indicate the distribution of several energy performance indices on the four prototype models, including the annual total site and source energy, annual cooling energy use (electricity), annual heating energy use (natural gas), peak electricity \& natural gas use. Key findings are summarized as follows:

(1) The impact of microclimate on the energy use of the HVAC systems is significant. Using different microclimate data can lead to as much as over $100 \%$ difference in annual heating energy use and $65 \%$ difference in annual cooling energy use.

(2) The impact of microclimate on the total annual site or source energy is much smaller. This is because (1) microclimate only affects HVAC energy use, which accounts for $20 \sim 25 \%$ of total energy use in the large office, and $40 \sim 50 \%$ in the large hotel. In this case, the relative impact on total energy use is reduced, (2) the heating demand and cooling demand compensate each other. For example, when cooling demand is increased under warmer weather, heating demand is decreased, so the overall impact is reduced.
(3) The impact of microclimate on building peak cooling and heating demand is significant, as much as a $30 \%$ difference in peak cooling electricity demand and over $100 \%$ difference in peak natural gas demand. This is critical from the supply-side perspective, as it will directly affect the required utility generation capacity. The impact on the total building peak demand is a bit less than the peak HVAC demand because of other end uses (e.g., lighting and plug loads).

(4) The impact of microclimate on energy performance varies with building types and vintages. Cooling and heating loads mainly consist of (1) heat gains through the envelope, mechanical ventilation, and infiltration, which microclimate has an impact on, and (2) heat gains from other sources, such as occupant, lighting and plug load, which barely change with climate. The variation of the absolute values of energy performance is purely affected by the former heat gains. On the other hand, the percentage difference is affected by the former heat gains, the proportion of former heat gains in the total load, and the baseline level. All the above factors vary with building types and vintages, resulting in different levels of microclimate impact on building energy performance.

In summary, microclimate data may be optional for estimating the total building energy consumption, but is crucial for more accurate estimation of the cooling and heating energy use, and more importantly, the peak demand, especially from the perspective of the utility supply side.

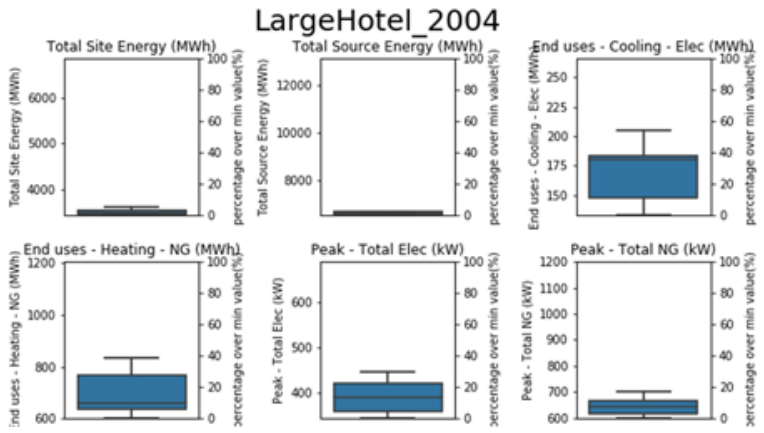

Figure 7: Box-whisker plot of energy performance index for the large hotel at the 2004 vintage.

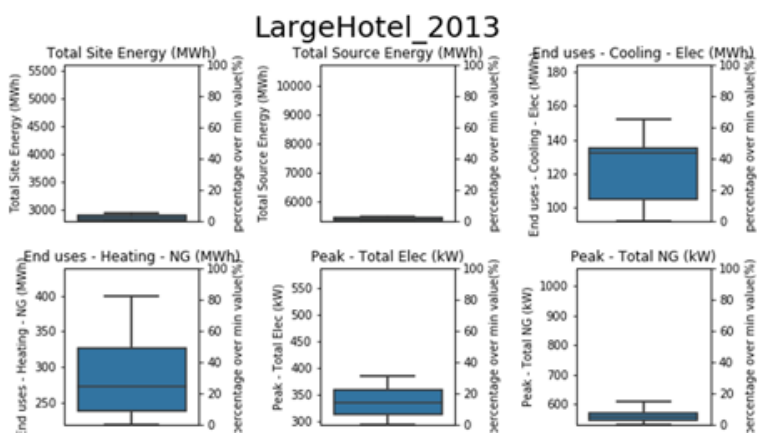

Figure 8: Box-whisker plot of energy performance index for the large hotel at the 2013 vintage. 


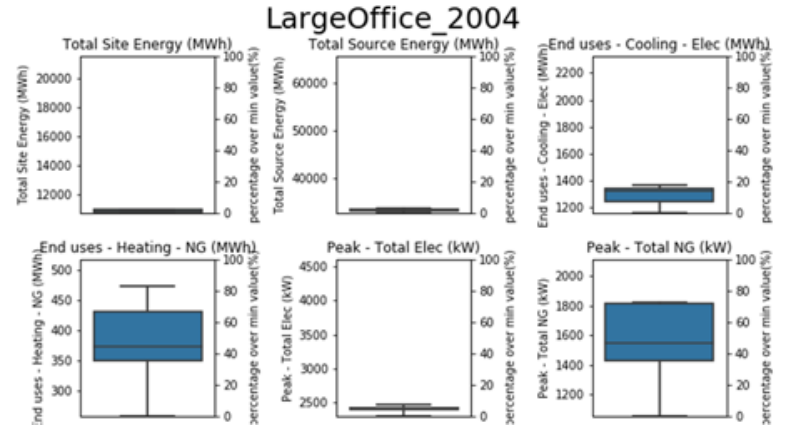

Figure 9: Box-whisker plot of energy performance index for the large office at the 2004 vintage.

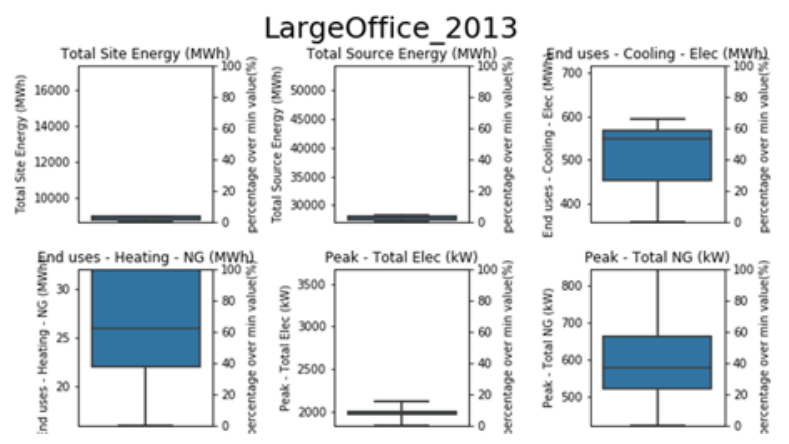

Figure 10: Box-whisker plot of energy performance index for the large office at the 2013 vintage.

\section{CONCLUSIONS}

A visualization of urban microclimate feature is developed in CityBES. San Francisco microclimate variations are significant and should be considered in building energy codes and standards as well as heat resilience policymaking. Local weather data should be used in building energy modeling to estimate building energy use. Future work will expand the coverage for other cities.

\section{ACKNOWLEDGMENTS}

We thank San Francisco the Department of Energy for providing the weather data. This work was also supported by the Assistant Secretary for Energy Efficiency and Renewable Energy, Office of Building Technologies of the United States Department of Energy, under Contract No. DE-AC02-05CH11231.

\section{REFERENCES}

[1] Hong, T., Chen, Y., Lee, S. H., \& Piette, M. A. (2016). CityBES: A web-based platform to support city-scale building energy efficiency. Urban Computing, 14.

[2] WBT. (2007). White Box Technologies Weather Data. Retrieved July 8, 2019, from http://weather.whiteboxtechnologies.com/.

[3] U.S. Department of Energy. (2019). Auxiliary Programs (p. 259). Retrieved from https://energyplus.net/sites/all/modules/custom/nrel_custom/pdfs/pdfs_v9.1.0/ AuxiliaryPrograms.pdf

[4] NOAA/ National Weather Service. (2014, May 28). Heat Index Equation. $\begin{array}{lccc}\text { Retrieved July } & 2, & 2019, \\ \text { https://www.wpc.ncep.noaa.gov/html/heatindex } & \text { equation.shtml }\end{array}$

[5] Dean, W. (2015). Creating and Mapping an Urban Heat Island Index for California. CalEPA.

[6] Yang, L., Jin, S., Danielson, P., Homer, C., Gass, L., Case, A., Costello, C., Dewitz, J., Fry, J., Funk, M., Grannemann, B., Rigge, M. and G. Xian. 2018. A New Generation of the United States National Land Cover Database: Requirements, Research Priorities, Design, and Implementation Strategies, p. $108-123$.

[7] R. Kyle Bocinsky (2019). FedData: Functions to Automate Downloading Geospatial Data Available from Several Federated Data Sources. R package version 2.5.7. https://CRAN.R-project.org/package=FedData

[8] Robert J. Hijmans (2019). raster: Geographic Data Analysis and Modeling. R package version 2.9-5. https://CRAN.R-project.org/package=raster

[9] Pebesma, E.J., 2004. Multivariable geostatistics in S: the gstat package. Computers \& Geosciences, 30: 683-691.

[10] Pebesma, E., 2018. Simple Features for R: Standardized Support for Spatial Vector Data. The R Journal 10 (1), 439-446, https://doi.org/10.32614/RJ-2018-009

[11] Leaflet contributors. (2019). Leaflet - a JavaScript library for interactive maps. Retrieved July 9, 2019, from https://leafletjs.com/index.html

[12] dygraphs contributors. (2014, September 18). dygraphs. Retrieved July 9, 2019, from http://dygraphs.com/

[13] Plotly contributors. (2019, March 3). Modern Analytic Apps for the Enterprise Plotly. Retrieved July 9, 2019, from https://plot.ly/

[14] Saito, T., Miyamura, H. N., Yamamoto, M., Saito, H., Hoshiya, Y., \& Kaseda, T. (2005, October). Two-tone pseudo coloring: Compact visualization for onedimensional data. In IEEE Symposium on Information Visualization, 2005. INFOVIS 2005. (pp. 173-180). IEEE.

[15] U.S. Department of Energy. (2013). Commercial Prototype Building Models. Retrieved https://www.energycodes.gov/development/commercial/prototype models

[16] D.B. Crawley, L.K. Lawrie, F.C. Winkelmann, W.F. Buhl, Y.J. Huang, C.O. Pedersen, et al., EnergyPlus: creating a new-generation building energy simulation program, Energy Build. 33 (2001) 319-331, http://dx.doi.org/10 1016/S0378-7788(00)00114-6. 